\title{
Survival probability of a diffusing test particle in a system of coagulating and annihilating random walkers.
}

\author{
R. Rajesh 1 , and Oleg Zaboronski ${ }^{2}$, , \\ ${ }^{1}$ Martin Fisher School of Physics, Brandeis University, Mailstop 057, Waltham, MA 02454-9110, USA \\ ${ }^{2}$ Mathematics Institute, University of Warwick, Gibbet Hill Road, Coventry CV4 7AL, UK
}

\begin{abstract}
We calculate the survival probability of a diffusing test particle in an environment of diffusing particles that undergo coagulation at rate $\lambda_{c}$ and annihilation at rate $\lambda_{a}$. The test particle dies at rate $\lambda^{\prime}$ on coming into contact with the other particles. The survival probability decays algebraically with time as $t^{-\theta}$. The exponent $\theta$ in $d<2$ is calculated using the perturbative renormalization group formalism as an expansion in $\epsilon=2-d$. It is shown to be universal, independent of $\lambda^{\prime}$, and to depend only on $\delta$, the ratio of the diffusion constant of test particles to that of the other particles, and on the ratio $\lambda_{a} / \lambda_{c}$. In 2-dimensions we calculate the logarithmic corrections to the power law decay of the survival probability. Surprisingly, the log corrections are non-universal. The 1-loop answer for $\theta$ in one dimension obtained by setting $\epsilon=1$ is compared with existing exact solutions for special values of $\delta$ and $\lambda_{a} / \lambda_{c}$. The analytical results for the logarithmic corrections are verified by Monte Carlo simulations.
\end{abstract}

PACS numbers: 05.10.Cc, 05.70.Ln, 82.40.Qt

\section{INTRODUCTION}

The calculation of the survival probability of a test particle in reaction diffusion systems has been studied in different contexts such as site persistence [1], walker persistence problems [2, 3, 4, 5, 6], polydispersity exponents in models of aggregation [6, 7, 8, 9] and predator-prey models [10, 11]. The approach to these problems have mostly been based on studying exactly solvable limiting cases or using mean field approximation and its improvements such as the Smoluchowski approximation [12, 13]. In recent years, field theoretic methods have proved successful in providing a general framework to understand these problems. In particular, the renormalization group analysis has been instrumental both in identifying the universal persistent features of reaction-diffusion systems and in extracting quantitative results about persistence exponents which could not be obtained using other methods 2, 6, 9, 11, 14, 15, 16]. In this paper, we apply field theoretic methods to the problem of the survival probability of diffusing test $B$-particles in a background of diffusing $A$-particles undergoing the reactions

$$
\begin{aligned}
& A+A \stackrel{\lambda_{c}}{\longrightarrow} A, \\
& A+A \stackrel{\lambda_{a}}{\longrightarrow} \emptyset, \\
& A+B \stackrel{\lambda^{\prime}}{\longrightarrow} A .
\end{aligned}
$$

The above reaction has been studied in the context of persistence. In one dimension and when $B$-particles are stationary, calculating the survival probability of $B$ particles is equivalent to calculating the fraction of spins that have not flipped up to time $t$ in the $q$-state Potts

\footnotetext{
*Electronic address: rrajesh@brandeis.edu
}

†Electronic address: olegz@maths.warwick.ac.uk model evolving via zero temperature Glauber dynamics, where $q=\frac{\lambda_{c}}{\lambda_{c}}+2$ 1]. The more general problem in which the $B$-particles are mobile with a diffusion constant equal to $\delta$ times the diffusion constant of the $A$-particles has been studied in Refs. 2, 3, 6, 17]. The density of $B$ particles then decays with time as $t^{-\theta(\delta, Q)}$, where $Q=$ $\left(\lambda_{c}+\lambda_{a}\right) /\left(\lambda_{c}+2 \lambda_{a}\right)$. As $Q$ varies from $1 / 2$ to 1 , the ratio $\lambda_{c} / \lambda_{a}$ varies from 0 to $\infty$. The known results for $\theta(\delta, Q)$ are briefly reviewed below.

When the dimension $d$ is greater than the upper critical dimension- two in this case- the decay exponents are obtained by solving the mean field rate equations with appropriately renormalized lattice-dependent reaction rate. In dimensions $d \leq 2$, fluctuation effects become important, and $\theta(\delta, Q)$ is no longer given by the rate equations. Exact solutions are one way of calculating exponents in 1-dimension. When $\delta=0$, by mapping the calculation of the persistence probability to a calculation of empty interval probabilities in the $A+A \rightarrow A$ model, it was shown that 1]

$$
\theta(0, Q)=\frac{2}{\pi^{2}}\left[\cos ^{-1}\left(\frac{1-2 Q}{\sqrt{2}}\right)\right]^{2}-\frac{1}{8}, \quad d=1 .
$$

Attempts to generalize the methods used in Ref. 1] to arbitrary $\delta$ were successful only in determining the values of $\left.\frac{d \theta}{d Q}\right|_{Q=0}$ and $\left.\frac{d \theta}{d \delta}\right|_{\delta=0}[3]$. Another solvable limit is $Q=$ 1 , when annihilation is absent. In this case, the problem reduces to a three particle problem which can be solved exactly to yield [18]

$$
\theta(\delta, 1)=\frac{\pi}{2 \cos ^{-1}[\delta /(1+\delta)]}, \quad d=1 .
$$

More general ways of understanding the effects of fluctuation in low dimensional reaction-diffusion systems include the Smoluchowski approximation 12, 13. which effectively reduces to the replacement of the reaction rates in the rate equations by diffusion-renormalized values, 
and the renormalization group formalism. The exponent $\theta(\delta, 1 / 2)$ was calculated using the Smoluchowski approximation in Ref. 17]. The advantage of Smoluchowski approximation is its computational simplicity. However, it is not clear how to improve this approximation in a systematic manner. Also, it was shown in Refs. 6, 9$]$ that while this approach gives an answer close to the actual one for $Q=1 / 2$, it becomes increasingly worse as $Q$ nears 1 . The field theoretic approach using the renormalization group formalism currently provides the only systematic way of calculating the decay exponents below the critical dimension. The exponent $\theta(\delta, 1 / 2)$ was calculated using field theoretic methods in Ref. 22. However, the renormalization group scheme used was complicated and did not capture the right logarithmic corrections (see Sec. IVB for a more detailed discussion). $\theta(1, Q)$ was calculated as an expansion in $(2-d)$ in [6, 9] in the context of domain wall persistence in the Potts model.

In this paper, we extend the formalism developed in Refs. 6, 9] to calculate $\theta(\delta, Q)$ for arbitrary $\delta$ and $Q$ to order $\epsilon$, where $\epsilon=2-d$. In particular we show that

$$
\begin{aligned}
\theta= & \frac{Q(1+\delta)}{2} \times \\
& {\left[2-\epsilon\left\{\frac{3}{2}+\ln \frac{1+\delta}{2}+\frac{Q(1+\delta) f(\delta)}{2}\right\}+O\left(\epsilon^{2}\right)\right] }
\end{aligned}
$$

where

$$
f(\delta)=1-2 \delta+2 \delta \ln \left(\frac{2}{1+\delta}\right)+\left(1-\delta^{2}\right) \int_{\frac{\delta-1}{\delta+1}}^{1} d u \frac{\ln (1-u)}{u} .
$$

The function $f(\delta)$ increases from $\left(1-\pi^{2} / 4\right)$ to 0 as $\delta$ increases from 0 to $\infty$. In 2 -dimensions, we calculate logarithmic corrections to the power law decay and show that

$$
\langle b\rangle \sim t^{-Q(1+\delta)} \ln (t)^{\alpha / 2},
$$

where $\langle b\rangle$ is the mean density of $B$-particles and

$$
\begin{aligned}
\alpha= & Q(1+\delta)\left[3+Q(1+\delta) f(\delta)+2 \ln \frac{1+\delta}{2}\right] \\
& +4 \pi Q(1+\delta)^{2}\left(\frac{1}{\lambda^{\prime}}-\frac{2}{(1+\delta)\left(\lambda_{a}+\lambda_{c}\right)}\right),
\end{aligned}
$$

with the function $f$ as defined in Eq. (5). A surprising feature of Eq. (7) is its non-universality for finite reaction rates, $\lambda, \lambda^{\prime}<\infty$. In this case $\alpha$ explicitly depends on both reaction rates. This is contrary to the usual belief that below the upper critical dimension the kinetics is diffusion limited and hence one may set reaction rates to infinite. Most exact solutions make use of this simplifying assumption. The above result serves as a counter example.

The rest of the paper is organized as follows. In Sec. II the model is defined. In Sec. III the rate equation approach is compared with the Smoluchowski approximation. The survival probability is calculated to one loop precision. In Sec. IV the renormalization group analysis of the problem is carried out and Eqs. (4), (6), and (7) are derived. The one-loop answer for $\theta$ is compared with the result of Smoluchowski approximation and also with known exact results in 1-dimension for special values of $\delta$ and $Q$. We also compare the analytical results with the results from numerical simulations. First, the predictions for the logarithmic corrections to the power law decay are confirmed numerically in the limit of instantaneous reactions. Second, the non-universality of logarithmic corrections for finite reaction rates is verified. Finally, we end with a summary and conclusions in Sec. $\mathbf{\nabla}$

\section{THE MODEL.}

Consider a $d$-dimensional hyper-cubic lattice whose sites may be occupied by $A$-particles and $B$-particles. Multiple occupancy of a site is allowed. Given a configuration of particles, the system evolves in time as follows. (i) At rate $D /(2 d)$, an $A$-particle hops to a nearest neighbor site. (ii) At rate $\delta D /(2 d)$, a $B$-particle hops to a nearest neighbor site. (iii) At rate $\lambda_{a}$, two $A$-particles at the same site annihilate each other. (iv) At rate $\lambda_{c}$, two $A$-particles at the same site coagulate together, thus reducing the number of $A$ particles by one. (v) At rate $\lambda^{\prime}$, a $B$-particle is absorbed by an $A$-particle at the same site. The initial number of $A$ - $(B-)$ particles at the lattice sites are chosen independently from a Poisson distribution with intensity $a_{0}\left(b_{0}\right)$.

The action corresponding to the continuous limit of the model can be derived from the master equation using Doi's formalism 19, 20, 21]. We skip the derivation and give the final result. The action is

$$
\begin{aligned}
S= & \int d t \int d^{d} x\left(\bar{a}\left(\partial_{t}-\nabla^{2}\right) a+\bar{b}\left(\partial_{t}-\delta \nabla^{2}\right) b+\frac{\lambda}{2 Q} \bar{a} a^{2}\right. \\
& \left.+\frac{\lambda}{2} \bar{a}^{2} a^{2}+\lambda^{\prime} \bar{b} a b+\lambda^{\prime} \bar{a} \bar{b} a b-\left(\bar{a} a_{0}+\bar{b} b_{0}\right) \delta(t)\right)
\end{aligned}
$$

where the $a$ and $b$ are complex fields corresponding to $A$ and $B$-particles, the diffusion constant $D$ has been set equal to 1 , and

$$
\begin{aligned}
Q & =\frac{\lambda_{c}+\lambda_{a}}{\lambda_{c}+2 \lambda_{a}}, \\
\lambda & =\lambda_{a}+\lambda_{c} .
\end{aligned}
$$

The knowledge of all the correlation functions of the fields $a, b$ allows one to construct all the correlation functions of local densities of $A$ and $B$ particles [22]. In particular, the mean density of $A$ and $B$ particles at $(\vec{x}, t)$ is equal to $\langle a(\vec{x}, t)\rangle$ and $\langle b(\vec{x}, t)\rangle$ respectively, where $\langle\ldots\rangle$ denotes functional average with respect to the functional measure Eq. (8).

The action can be brought into a more convenient form by rescaling the fields as follows: $(\bar{a}, \bar{b}) \rightarrow Q^{-1}(\bar{a}, \bar{b})$, 


$$
\begin{aligned}
& \left(a_{0}, b_{0}\right) \rightarrow Q\left(a_{0}, b_{0}\right), \text { and }\left(\lambda, \lambda^{\prime}\right) \rightarrow 2 Q\left(\lambda, \lambda^{\prime}\right) \text {. Then } \\
& \begin{aligned}
S & =\int d t \int d^{d} x\left(\bar{a}\left(\partial_{t}-\nabla^{2}\right) a+\bar{b}\left(\partial_{t}-\delta \nabla^{2}\right) b+\lambda \bar{a} a^{2}\right. \\
& \left.+\lambda \bar{a}^{2} a^{2}+2 \lambda^{\prime} Q \bar{b} a b+2 \lambda^{\prime} \bar{a} \bar{b} a b-\left(\bar{a} a_{0}+\bar{b} b_{0}\right) \delta(t)\right)(11)
\end{aligned}
\end{aligned}
$$

The Feynman diagrams corresponding to the action in Eq. (11) are shown in Fig. [1]

We are interested in the mean density of $B$-particles in the limit of large time, as the survival probability is proportional to mean density. The evolution of mean density of $A$-particles $\langle a\rangle$ is independent of the statistics of $B$-particles and decays at large times $t$ as [14]

$$
\langle a\rangle \sim \begin{cases}t^{-d / 2}, & d<2 \\ t^{-1} \ln (t), & d=2 \\ t^{-1}, & d>2\end{cases}
$$

\section{THE COMPUTATION OF PERSISTENCE EXPONENT USING MEAN FIELD AND SMOLUCHOWSKI APPROXIMATIONS.}

The perturbative expansion of $\langle b\rangle$ in powers of $\lambda$ can be constructed using the Feynman diagrams shown in Fig. 10 23]. Diagrammatically, $\langle a\rangle(\langle b\rangle)$ is the sum of all Feynman diagrams with one outgoing $a$-(b-)line respectively. Clearly, there is an infinite number of diagrams contributing to $\langle a\rangle$ and $\langle b\rangle$. These diagrams can be grouped together according to the number of loops that they contain, thus giving rise to the loop expansion. Let $\epsilon=2-d$. A simple combinatorial argument shows that the contribution from a diagram with $n$ loops is proportional to $g(t)^{n}$, where $g(t)=\lambda t^{\epsilon / 2}[15]$. When $\epsilon<0$, the main contribution to $\langle a\rangle$ and $\langle b\rangle$ comes from properly renormalized tree level diagrams (diagrams without loops). When $\epsilon>0$, the loop expansion fails since for large times $g(t)$ is no longer a small perturbation parameter. We therefore conclude that 2 is the upper critical dimension. For $d<2$ we will use the formalism of perturbative renormalization group to convert the loop expansion into an $\epsilon$-expansion and calculate scaling exponents as a series in $\epsilon$.

\section{A. Tree level diagrams}

In $d<2$ and small times, tree diagrams give the main contribution to the survival probability. Let $\langle a\rangle_{\mathrm{mf}}$ and $\langle b\rangle_{\mathrm{mf}}$ be mean field densities given by the sum of contributions coming from tree diagrams with a single outgoing $a$-line and $b$-line respectively. We denote $\langle a\rangle_{\mathrm{mf}}$ and $\langle b\rangle_{\mathrm{mf}}$ by thick solid lines and thick dashed lines respectively. The integral equations satisfied by $\langle a\rangle_{\mathrm{mf}}$ and $\langle b\rangle_{\mathrm{mf}}$ are presented in diagrammatic form in Fig. 22 a) and 2 (b). After differentiating with respect to time, they can be
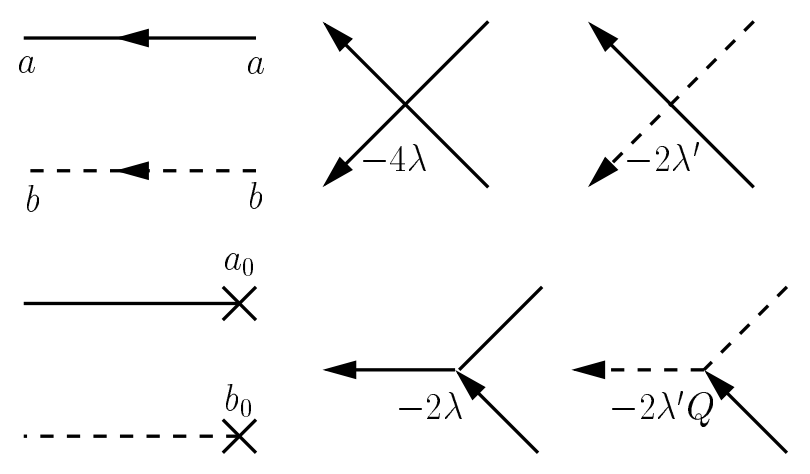

FIG. 1: Propagators and vertices of the theory.

(a)

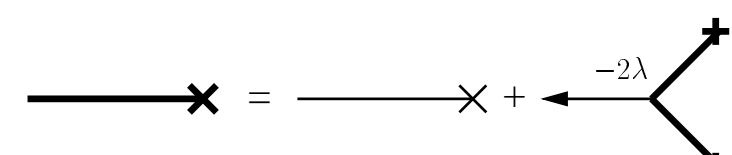

(b)

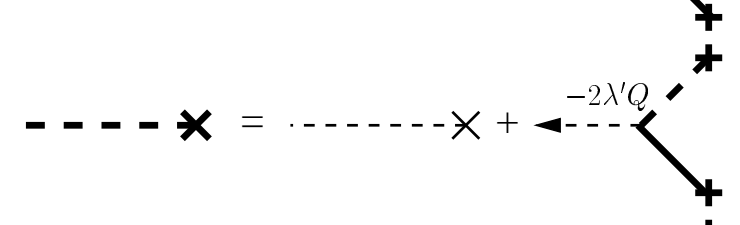

(c)

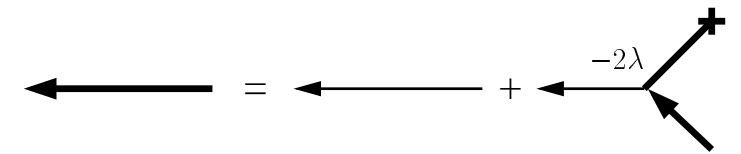

(d)

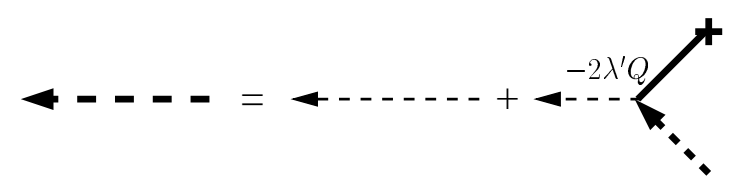

FIG. 2: Diagrammatic form of mean field equations for (a) mean particle density $\langle b\rangle$, (b) mean density of $B$-particles $\langle b\rangle$, (c) $G_{\mathrm{mf}}^{\mathrm{NN}}$, and (d) $G_{\mathrm{mf}}^{\mathrm{PP}}$.

written in analytic form as

$$
\begin{aligned}
\partial_{t}\langle a\rangle & =-\lambda\langle a\rangle^{2}, \\
\partial_{t}\langle b\rangle & =-2 Q \lambda^{\prime}\langle b\rangle\langle a\rangle,
\end{aligned}
$$

in which one can easily recognize the rate equations of the model. Thus, the identification of tree-level truncation with mean field approximation is justified.

Equations (13) and (14) are easily solved yielding

$$
\begin{aligned}
\langle a(t)\rangle_{\mathrm{mf}} & =\frac{a_{0}}{1+\lambda a_{0} t}, \\
\langle b(t)\rangle_{\mathrm{mf}} & =\frac{b_{0}}{\left(1+\lambda a_{0} t\right)^{2 Q \lambda^{\prime} / \lambda}},
\end{aligned}
$$

where $a_{0}$ and $b_{0}$ are the initial densities of $A$ - and $B$ particles respectively. Thus,

$$
\theta(\delta, Q)=2 Q \frac{\lambda^{\prime}}{\lambda}, \quad d>2 .
$$

The result is explicitly dependent on $\lambda^{\prime}, \lambda$ while being independent of $\delta$ and describes the reaction-limited regime 
of the problem. It should be mentioned here that the above result is valid only in the limit when the reaction rates are the smallest parameters in the problem, i.e. $\lambda, \lambda^{\prime} \ll l_{0}^{d-2}$, where $l_{0}$ is the lattice spacing. In the other limit when the lattice spacing is the smallest parameter in the problem, the exponents gets modified to [24]

$$
\theta(\delta, Q)=Q(1+\delta), \quad l_{0}^{d-2} \ll \lambda, \lambda^{\prime}, \quad d>2 .
$$

In order to estimate the validity of the mean field approximation in $d \leq 2$, the one-loop corrections to the mean field answer have to be evaluated. In calculating loop corrections to Eqs. (15) and (16), we are faced with the problem of summing over infinite number of diagrams containing a given number of loops. This problem can be simplified by introducing mean field propagators which are sums of all tree diagrams with one incoming line and one outgoing line. Expressed in terms of these mean field propagators, there are only a finite number of diagrams with a fixed number of loops.

Let $G_{\mathrm{mf}}^{\mathrm{NN}}$ and $G_{\mathrm{mf}}^{\mathrm{PP}}$ be mean field propagators. The integral equations satisfied by them are presented in diagrammatic form in Figs. 2(c) and2/(d). The solutions to these equations are

$$
\begin{aligned}
& G_{\mathrm{mf}}^{\mathrm{NN}}(\mathbf{2} \mid \mathbf{1})=\left(\frac{\left.\left\langle a\left(t_{2}\right)\right\rangle_{\mathrm{mf}}\right)}{\left.\left\langle a\left(t_{1}\right)\right\rangle_{\mathrm{mf}}\right)}\right)^{2} G_{1}(\mathbf{2} \mid \mathbf{1}), \\
& G_{\mathrm{mf}}^{\mathrm{PP}}(\mathbf{2} \mid \mathbf{1})=\left(\frac{\left\langle a\left(t_{2}\right)\right\rangle_{\mathrm{mf}}}{\left\langle a\left(t_{1}\right)\right\rangle_{\mathrm{mf}}}\right)^{2 Q \lambda^{\prime} / \lambda} G_{\delta}(\mathbf{2} \mid \mathbf{1}),
\end{aligned}
$$

where $\mathbf{1}=\left(\vec{x}_{1}, t_{1}\right), \mathbf{2}=\left(\vec{x}_{2}, t_{2}\right)$ and $G_{D}$ is the Green's function of the linear diffusion equation with diffusion constant $D$.

\section{B. Smoluchowski approximation}

Before presenting the renormalization group calculation of $\theta(\delta, Q)$, we briefly discuss a method commonly used to study fluctuation effects in reaction-diffusion systems, namely the Smoluchowski approximation. The essential idea of the Smoluchowski approach is to relate the reaction rates $\lambda$ and $\lambda^{\prime}$ to the diffusion rates. One assumes that particles react instantaneously when they come within a fixed radius of each other (see 2, 17] for a more detailed discussion). Knowing the first return probabilities of random walks, one obtains for $d<2$,

$$
\begin{aligned}
\lambda & \sim \text { const } \times \mathrm{t}^{\mathrm{d} / 2-1}, \\
\lambda^{\prime} & \sim \text { const } \times\left(\frac{1+\delta}{2}\right)^{\mathrm{d} / 2} \mathrm{t}^{\mathrm{d} / 2-1} .
\end{aligned}
$$

In $d=2$ additional log corrections appear and

$$
\begin{aligned}
\lambda & \sim \frac{\text { const }}{\ln (t)} \\
\lambda^{\prime} & \sim \frac{\text { const } \times(1+\delta)}{2 \ln \left[\left(\frac{1+\delta}{2}\right) t\right]} .
\end{aligned}
$$

Replacing $\lambda$ and $\lambda^{\prime}$ in Eqs. (13) and (14) by the effective reaction reaction rates, and solving for $\langle b\rangle$, we obtain

$$
\langle b\rangle_{S} \sim \begin{cases}t^{-d Q\left(\frac{1+\delta}{2}\right)^{d / 2}}, & d<2, \\ \left(\frac{\ln (t)}{t}\right)^{Q(1+\delta)}(\ln (t))^{Q(1+\delta) \ln [(1+\delta) / 2]}, & d=2 .\end{cases}
$$

where $\langle b\rangle_{S}$ denotes the mean density of $B$-particles obtained from Smoluchowski approximation. In particular, the Smoluchowski theory's prediction for $\theta$ is

$$
\theta_{S}(\delta, Q)=d Q\left(\frac{1+\delta}{2}\right)^{d / 2}, d<2 .
$$

This answer for $\theta$ depends on $\delta, Q$ and space dimensionality $d$. It does not however depend on $\lambda$ and $\lambda^{\prime}$. Thus, unlike the mean field answer Eq. (17), it has the correct universality properties for a quantity describing reaction-diffusion systems in the diffusion-limited regime. However, the Smoluchowski result differs considerably from the correct result when $Q$ nears 1 . For example, in one dimension $\theta_{S}(1,1)=1.0$ while $\theta(1,1)=1.5$ (see Eq. (3)). For more comparisons, see Sec. IVA It is not clear how one can improve the Smoluchowski approximation. The renormalization group formalism, though more involved, provides a systematic way to go beyond the Smoluchowski approximation.

\section{One loop diagrams}

The rate equation results do not depend on the diffusion coefficients of the particles or the dimensionality of the ambient space. These parameters appear in the one-loop corrections to the tree level answers. Using the mean field propagators and densities, it is easy to classify all the one loop diagrams contributing to $\langle b\rangle$. These are shown in Fig. 3. Skipping the computations we present the final answers for contributions corresponding to each of the Feynman diagrams in the limit $a_{0} \rightarrow \infty$ :

$$
\begin{aligned}
& (a)=\frac{32 Q \lambda^{\prime 2} b_{0} t^{\epsilon / 2}}{\lambda\left(a_{0} \lambda t\right)^{2 Q \lambda^{\prime} / \lambda}[4 \pi(1+\delta)]^{d / 2} \epsilon^{2}(\epsilon+2)} \\
& (b)=\frac{8 Q^{2} \lambda^{\prime 2} b_{0}(1+\delta) t^{\epsilon / 2}}{\lambda\left(a_{0} \lambda t\right)^{2 Q \lambda^{\prime} / \lambda}[4 \pi(1+\delta)]^{d / 2} \epsilon}[f(\delta)+O(\epsilon)](28) \\
& (c)=\frac{-256 Q \lambda^{\prime} b_{0} t^{\epsilon / 2}}{\left(a_{0} \lambda t\right)^{2 Q \lambda^{\prime} / \lambda}(8 \pi)^{d / 2} \epsilon^{2}(\epsilon+2)^{2}(\epsilon+4)}
\end{aligned}
$$

where $(a),(b)$ and $(c)$ refer to the contributions from diagrams in Figs. 3(a), 31(b) and 3(c) respectively, and

$f(\delta)=1-2 \delta+2 \delta \ln \left(\frac{2}{1+\delta}\right)+\left(1-\delta^{2}\right) \int_{\frac{\delta-1}{\delta+1}}^{1} d u \frac{\ln (1-u)}{u}$.

Adding these one-loop contributions to the mean field answer Eq. (16), we obtain in the limit $a_{0} \rightarrow \infty$,

$$
\langle b(t)\rangle=\frac{A}{t^{2 Q \lambda^{\prime} / \lambda}}\left[1+\frac{8 Q \lambda^{\prime} t^{\epsilon / 2}}{(4 \pi)^{d / 2} \epsilon}\left\{\frac{4 \lambda^{\prime}}{\lambda(1+\delta)^{d / 2}} \frac{1}{\epsilon(\epsilon+2)}\right.\right.
$$




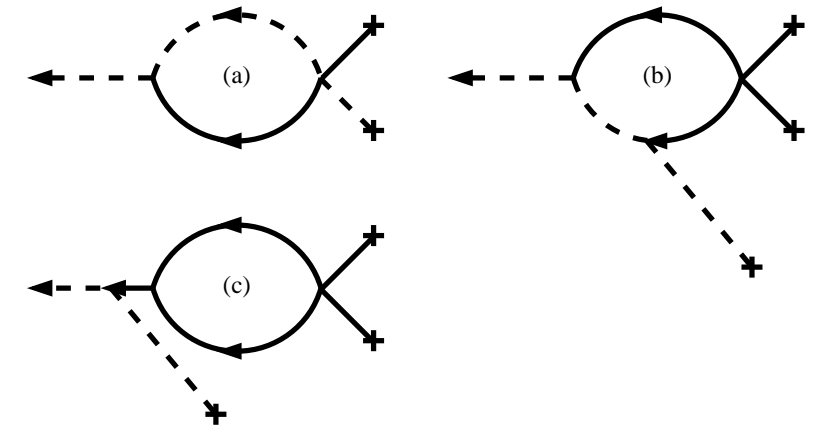

FIG. 3: One loop corrections to the mean field result for $\langle b\rangle$.

$$
\begin{aligned}
& \left.\left.-\frac{32}{2^{d / 2}} \frac{1}{\epsilon(\epsilon+2)^{2}(\epsilon+4)}+\frac{Q \lambda^{\prime}(1+\delta)^{\epsilon / 2} f(\delta)}{2 \lambda}\right\}\right] \\
& +2 \text { - and higher loop corrections, }
\end{aligned}
$$

where $A=b_{0} /\left(a_{0} \lambda\right)^{2 Q \lambda^{\prime} / \lambda}$. We see that if $\lambda \sim \lambda^{\prime}$, then the mean field answer Eq. (17) is correct in $d<2$ only if $Q \lambda t^{\epsilon / 2} \ll 1$. Clearly, this condition breaks down in the limit of large times in $d<2$.

\section{PERTURBATIVE COMPUTATION OF $\theta(\delta, Q)$ NEAR $d=2$ USING RG METHOD.}

In this section, we calculate the large time behavior of $\langle b\rangle$. The loop expansion for $\langle b(t)\rangle$ fails at large times in $d \leq 2$. To extract the large time behavior of $\langle b(t)\rangle$ in $d \leq 2$ we will use the formalism of perturbative renormalization group.

The renormalization group formalism used in Refs. 6 , 9] for the case $\delta=1$ was based on the Callan-Symanzik equations for the mean density of $B$-particles. There were two relevant couplings for the theory: the reaction rate $\lambda$ and the initial density $b_{0}$. The anomalous dimension of $\langle b(t)\rangle$ was attributed to the renormalization of $b_{0}$.

This approach turns out to be very complicated when $\delta \neq 1$. This is due to the explicit dependence of the classical scaling dimension of $\langle b(t)\rangle$ on $\lambda$ and $\lambda^{\prime}$. Further complications arise due to non-commutativity of $\epsilon \rightarrow 0$ and $a_{0} \rightarrow \infty$ limits, which leads to an apparent order$1 / \epsilon^{2}$ singularity in the one-loop correction to $\langle b(t)\rangle$ [see Eq. (31)].

These problems are circumvented by analyzing the large time asymptotic behavior of the logarithmic derivative of $\langle b(t)\rangle$, rather than $\langle b(t)\rangle$ itself. It follows from Eq. (31) that

$$
t \partial_{t}[\ln (\langle b(t)\rangle)]=\Pi(t)
$$

where

$$
\begin{aligned}
& \Pi(t)=-2 Q \frac{\lambda^{\prime}}{\lambda}+\frac{4 Q \lambda^{\prime} t^{\epsilon / 2}}{(4 \pi)^{d / 2}}\left\{\frac{4 \lambda^{\prime}}{\lambda(1+\delta)^{d / 2}} \frac{1}{\epsilon(\epsilon+2)}\right. \\
& \left.-\frac{2^{-d / 2} 32}{\epsilon(\epsilon+2)^{2}(\epsilon+4)}+\frac{Q \lambda^{\prime}(1+\delta)^{\epsilon / 2} f(\delta)}{2 \lambda}\right\}+O\left(\lambda^{2}\right) .
\end{aligned}
$$

The large time asymptotic behavior of $\Pi(t)$ can be obtained by solving the Callan-Symanzik equation with initial conditions given by the right-hand side of Eq. (33) regularized at some reference time $t_{0}$ (see Ref. 22] for a review). The coefficients of Callan-Symanzik equation are determined by the law of renormalization of all the relevant couplings of the theory. Power counting analogous to that carried out in Ref. [9] shows that there are only two relevant couplings of the theory which determine large time behavior of $\Pi(t)$ in $d \leq 2$ : the reaction rates $\lambda$ and $\lambda^{\prime}$. We mention here that $\Pi(t)$ is simply related to the polarization operator used in Ref. [9].

Let $g_{0}=\lambda t_{0}^{\epsilon / 2}, g_{0}^{\prime}=\lambda t_{0}^{\epsilon / 2}$ be the dimensionless reaction rates. We choose $t_{0}$ in such a way that $g_{0}, g_{0}^{\prime} \ll 1$. The law of renormalization of reaction rates has been worked out in Ref. 2, 14]. The renormalized reaction rates $g_{R}$ and $g_{R}^{\prime}$ are related to $g_{0}$ and $g_{0}^{\prime}$ as follows:

$$
\begin{aligned}
& g_{R}=\frac{g_{0}}{1+g_{0} / g_{*}}, \\
& g_{R}^{\prime}=\frac{g_{0}^{\prime}}{1+g_{0}^{\prime} / g_{*}^{\prime}} .
\end{aligned}
$$

Here $g_{*}$ and $g_{*}^{\prime}$ are the nontrivial fixed points of the renormalization group flow in the space of effective coupling constants, and are given by

$$
\begin{aligned}
& g_{*}=\frac{(8 \pi)^{d / 2}}{2 \Gamma(\epsilon / 2)}, \\
& g_{*}^{\prime}=\frac{[4 \pi(1+\delta)]^{d / 2}}{2 \Gamma(\epsilon / 2)},
\end{aligned}
$$

where $\Gamma(x)$ is the Euler Gamma function. The renormalization of both coupling constants is due to the same physical effect - the recurrence of random walks in $d \leq 2$.

$\Pi\left(t_{0}\right)$ expressed in terms of $g_{R}$ and $g_{R}^{\prime}$ has the following form:

$$
\begin{aligned}
\Pi\left(t_{0}\right)= & -2 Q \frac{g_{R}^{\prime}}{g_{R}}+\frac{Q g_{R}^{\prime}}{\pi}\left[\frac{g_{R}^{\prime}(\gamma-1)}{g_{R}(1+\delta)}+\frac{5-2 \gamma}{4}\right. \\
& \left.+\frac{Q g_{R}^{\prime} f(\delta)}{2 g_{R}}+O(\epsilon)\right]+O\left(g_{R}^{2}\right),
\end{aligned}
$$

where $\gamma$ is the Euler constant. $\Pi\left(t_{0}\right)$ regarded as a function of $g_{R}$ and $g_{R}^{\prime}$ is non-singular at $\epsilon=0$. As a result this expression is valid for $d \leq 2$. The lack of $t_{0}$-dependence of $\Pi(t)$ for $t>t_{0}$ is expressed by the following renormalization group (Callan-Symanzik) equation:

$$
\left.\left[t \frac{\partial}{\partial t}+\frac{\beta\left(g_{R}\right)}{2} \frac{\partial}{\partial g_{R}}+\frac{\beta\left(g_{R}^{\prime}\right)}{2} \frac{\partial}{\partial g_{R}^{\prime}}\right)\right] \Pi(t)=0
$$

where $\beta\left(g_{R}\right)=-2 t_{0} \partial g_{R} / \partial t_{0}$ and $\beta\left(g_{R}^{\prime}\right)=-2 t_{0} \partial g_{R}^{\prime} / \partial t_{0}$ are the beta functions,

$$
\begin{aligned}
& \beta\left(g_{R}\right)=\frac{g_{R}\left(g_{R}-g_{*}\right) \epsilon}{g_{*}}, \\
& \beta\left(g_{R}^{\prime}\right)=\frac{g_{R}^{\prime}\left(g_{R}^{\prime}-g_{*}^{\prime}\right) \epsilon}{g_{*}^{\prime}} .
\end{aligned}
$$


We will now solve Eq. (39) with the initial condition given by Eq. (38) to obtain the large time asymptotic behavior of $\Pi$. We then extract the large time asymptotic behavior of $\langle b(t)\rangle$ by solving Eq. (32).

\section{A. Survival probability in $d<2$}

At large times, the solutions of Callan-Symanzik equation (39) are governed by the stable fixed points of the beta functions. In $d<2$, these are $g_{R}=g_{*}$ and $g_{R}^{\prime}=g_{*}^{\prime}$. It then follows that

$$
\begin{aligned}
\Pi(t)= & -2 Q(1+\delta)+\epsilon Q(1+\delta)\left[\ln \frac{1+\delta}{2}+\frac{3}{2}\right. \\
& \left.+Q \frac{1+\delta}{2} f(\delta)\right]+O\left(\epsilon^{2}, t^{-\epsilon / 2}\right) .
\end{aligned}
$$

Substituting Eq. (42) into Eq. (32) and solving for $\langle b(t)\rangle$, we obtain the $O(\epsilon)$ result for $\theta$ :

$$
\begin{aligned}
\theta= & \frac{Q(1+\delta)}{2} \times \\
& {\left[2-\epsilon\left\{\frac{3}{2}+\ln \frac{1+\delta}{2}+\frac{Q(1+\delta) f(\delta)}{2}\right\}+O\left(\epsilon^{2}\right)\right](43) }
\end{aligned}
$$

where the function $f(\delta)$ is as in Eq. (30).

We now compare the 1-dimensional result obtained by putting $\epsilon=1$ in Eq. (43) with exact results in 1dimension for special values of $\delta$ and $Q$. The exact result for $\theta(0, Q)$ in 1-dimension is given in Eq. (2), while that for $\theta(\delta, 1)$ is given in Eq. (3). Figs 4 and 5 show the results for $\delta=0$ and $Q=1$ respectively. The $\epsilon$-expansion result is seen to compare very well with the exact result. On the other hand, the Smoluchowski results fail for $Q$ larger than $1 / 2$ when $\delta=0$, and for all $\delta$ when $Q=1$. It should be noted that when $\delta$ becomes large, the $\epsilon$-expansion should fail and Smoluchowski result should give a much better approximation.

\section{B. Survival probability in $d=2$.}

The upper critical dimension of our model is two. The non-trivial fixed points of the $\beta$-functions Eqs. (40) and (41) vanish at $d=2$. It is then expected that the rate equation results give the correct large time behavior of mean densities, perhaps modulo logarithmic corrections. This turns out to be incorrect. The complication comes from the fact that $\theta$ predicted by the mean field theory (see Eq. (17)) is non-universal and depends on the ratio of coupling constants $g_{R}$ and $g_{R}^{\prime}$. Each of these couplings is marginally relevant in two dimensions and flows under RG transformations to 0 as $[\ln (t)]^{-1}$. Their ratio flows to a finite universal value which determines the algebraic decay of the survival probability. However, the deviation from this universal value vanishes with time as $C / \ln (t)$, where $\mathrm{C}$ is a non-universal constant. This results in nonuniversal logarithmic corrections to the universal power

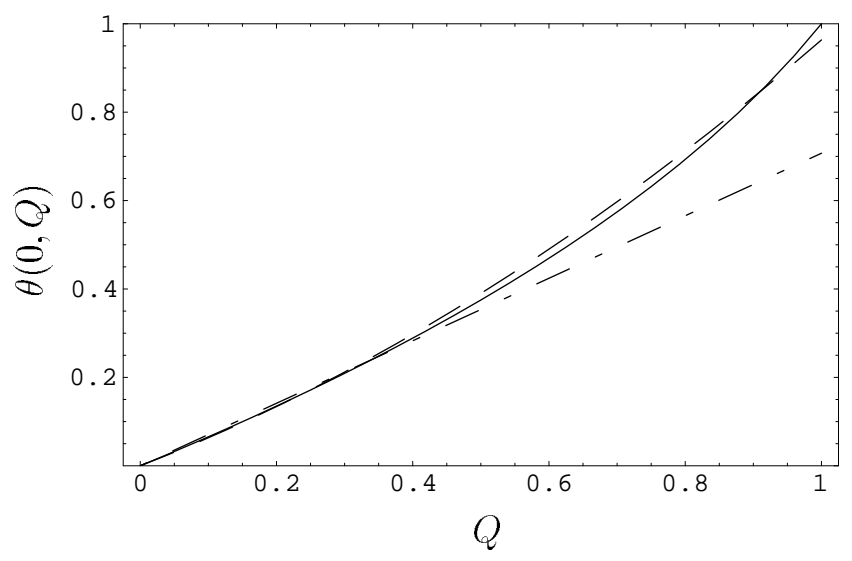

FIG. 4: The one-loop answer (Eq. (43)) is compared with exact result in 1-dimension when $\delta=0$ (Eq. (2)). The solid line corresponds to the exact answer, dashed line to one-loop, and dot dashed line to the Smoluchowski result (Eq. 25)).

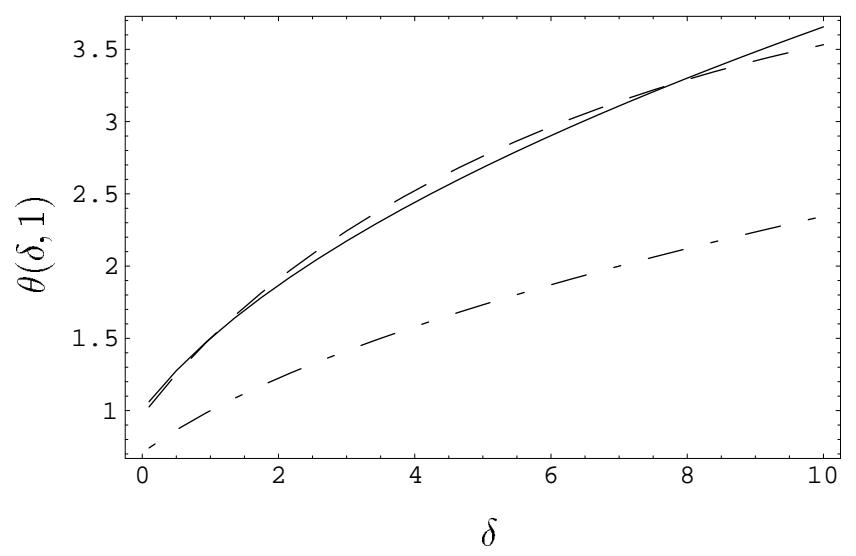

FIG. 5: The one-loop answer (Eq. (43) ) is compared with exact result in 1-dimension when $Q=1$ (Eq. (3)). The solid line corresponds to the exact answer, dashed line to one-loop, and dot dashed line to the Smoluchowski result (Eq. (25)).

law decay of survival probability. It is worth mentioning that in $d<2$, similar considerations lead to a conclusion that the amplitude of corrections to scaling is nonuniversal.

We need to solve the Callan-Symanzik equation (39) with coefficients calculated at $d=2$ :

$$
\begin{aligned}
\left.\beta(g)\right|_{d=2} & =\frac{g^{2}}{2 \pi}, \\
\left.\beta\left(g^{\prime}\right)\right|_{d=2} & =\frac{g^{\prime 2}}{\pi(1+\delta)} .
\end{aligned}
$$

Then Eq. (39) reduces to

$$
\left[t \frac{\partial}{\partial t}+\frac{g_{R}^{2}}{4 \pi} \frac{\partial}{\partial g_{R}}+\frac{g_{R}^{\prime 2}}{2 \pi(1+\delta)} \frac{\partial}{\partial g_{R}^{\prime}}\right] \Pi(t)=0
$$

which has to be solved with the initial condition given by 


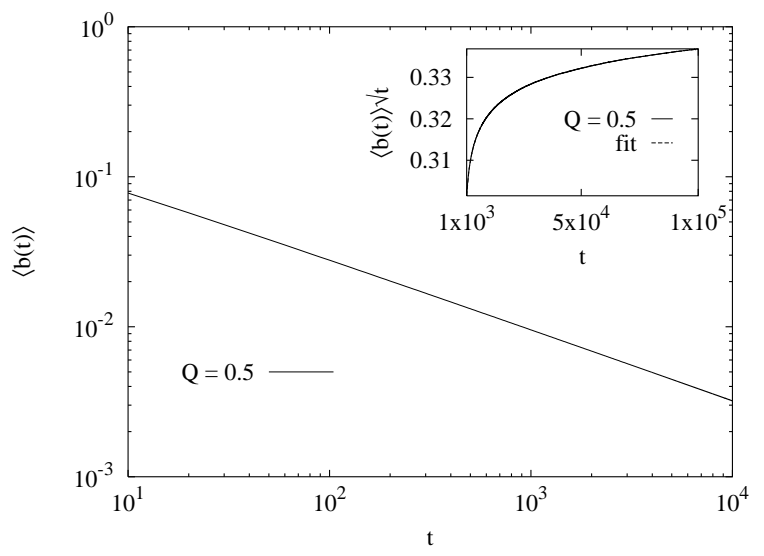

FIG. 6: The variation of the mean density of $B$-particles in two dimensions with time is shown. The simulations were done on a $3200 \times 3200$ lattice for $Q=0.5$ and $\delta=0$. The data has been averaged 1000 times. In the inset, the variation of $\langle b\rangle \sqrt{t}$ with time is shown. The power of the logarithm in the best fit is $0.23 \pm 0.03$.

Eq. (38) at $t=t_{0}, \epsilon=0$. The solution is

$$
\begin{aligned}
& \Pi(t)=-Q(1+\delta)+\frac{2 Q(1+\delta)}{\ln \left(t / t_{0}\right)}\left[\frac{3}{4}+\frac{Q}{2} f(\delta)\right. \\
& \left.+\pi(1+\delta)\left(\frac{1}{g_{R}^{\prime}}-\frac{2}{(1+\delta) g_{R}}\right)\right]+O\left(\frac{1}{\ln ^{2}(t)}\right)
\end{aligned}
$$

The non-universal term in Eq. (47) is proportional to $1 / g_{R}^{\prime}-2 /\left[(1+\delta) g_{R}\right]$. It is convenient to express this amplitude in terms of bare couplings. In two dimensions,

$$
\frac{1}{g_{R}^{\prime}}-\frac{2}{(1+\delta) g_{R}}=\frac{1}{g_{0}^{\prime}}-\frac{2}{(1+\delta) g_{0}}+\frac{\ln \left(\frac{1+\delta}{2}\right)}{2 \pi(1+\delta)} .
$$

In $d<2$, Eq. (48) has to be modified by the omitting the logarithmic term on the right hand side.

Solving Eq. (32) with Eq. (47) substituted for the right hand side and taking (48) into account, one finds that

$$
\langle b(t)\rangle=\text { const } \times \frac{\left(g_{R} \ln \left(t / t_{0}\right)\right)^{\alpha / 2}}{\left(g_{R} t\right)^{Q(1+\delta)}}\left(1+O\left(\frac{1}{\ln \left(t / t_{0}\right)}\right)\right),
$$

where

$$
\begin{aligned}
\alpha= & Q(1+\delta)\left[3+Q(1+\delta) f(\delta)+2 \ln \frac{1+\delta}{2}\right] \\
& +4 \pi Q(1+\delta)^{2}\left(\frac{1}{\lambda^{\prime}}-\frac{2}{(1+\delta) \lambda}\right) .
\end{aligned}
$$

Thus, in two dimensions, the power law exponent is universal and independent of $\lambda$ and $\lambda^{\prime}$. However, the logarithmic corrections are non-universal and depend on microscopic reaction rates $\lambda^{\prime}=g_{0}^{\prime}$ and $\lambda=g_{0}$. However, most simulations are done in the limit when the reactions are instantaneous. In this limit, the non-universal term in Eq. (50) is zero.

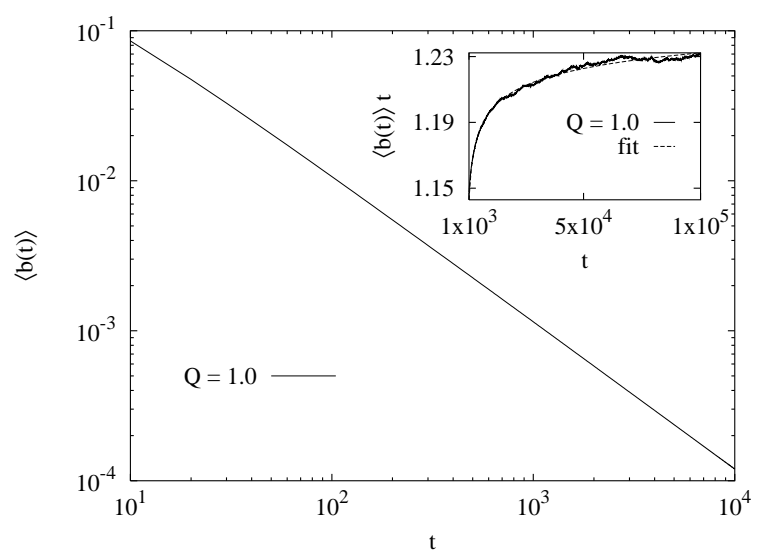

FIG. 7: The variation of the mean density of $B$-particles in two dimensions with time is shown. The simulations were done on a $3200 \times 3200$ lattice for $Q=1.0$ and $\delta=0$. The data has been averaged 1000 times. In the inset, the variation of $\langle b\rangle t$ with time is shown. The power of the logarithm in the best fit is $0.08 \pm 0.04$.

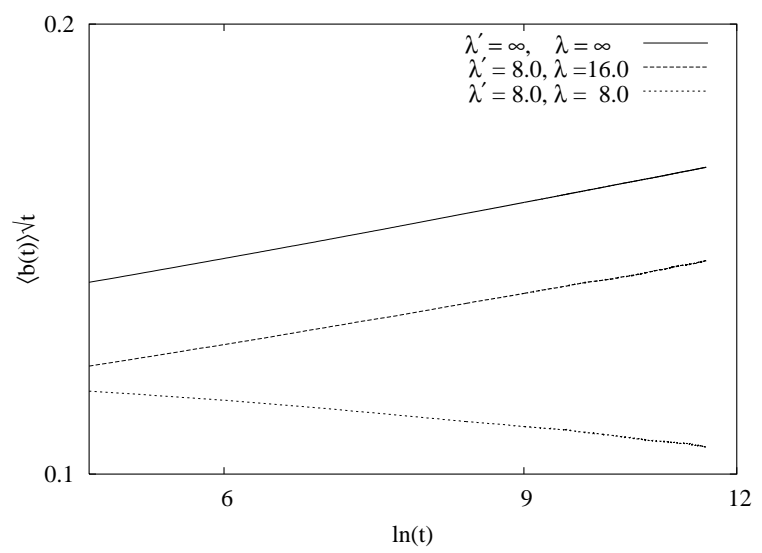

FIG. 8: The variation of the mean density of $B$-particles in two dimensions with time is shown. The simulations were done on a $900 \times 900$ lattice for $Q=0.5$ and $\delta=0$. The data has been averaged 1000 times.

The log corrections in Eq. (50) are different from the $\log$ corrections calculated for the $Q=1 / 2$ case in Ref. [2]. This discrepancy is due to the fact that only renormalized tree level computations were done in [2], while to obtain the correct logarithmic dependence, one-loop corrections have to be taken into account.

We also mention that if one were to ignore the contribution from one-loop diagrams, then the log corrections would be identical with the log corrections obtained from the Smoluchowski approximation [see Eq. (25)], and will be different from the log corrections obtained from renormalized tree-level as in Ref. 2].

We now study logarithmic corrections numerically. First, consider the case when the microscopic reactions are instantaneous, i.e., $\lambda=\lambda^{\prime}=\infty$. In this limit, the non-universal term in Eq. (50) is equal to zero. The 
Monte Carlo simulations were done for this case on a two dimensional lattice of size $3200 \times 3200$ with periodic boundary conditions. As the reactions are instantaneous, the maximum number of particles at a site is one. The simulations were done for $\delta=0$, i.e., immobile $B$ particles. The results for $Q=0.5$ and $Q=1.0$ are shown in Figs. [6] and 7 respectively. For $Q=0.5, \alpha=0.23 \pm 0.03$ which compares well with the theoretical value of approximately 0.22 in Eq. (50). Note that renormalized tree-level answer is $\alpha=0.5$ [2]. For $Q=1.0$, the numerical value is $.08 \pm 0.04$, which compares well with the theoretical value of approximately 0.07 . This answer also deviates significantly from renormalized tree-level value of 1.0 .

Second, we study the logarithmic corrections for finite reaction rates to test the non-universal term in Eq. (50). In this case the lattice size is $900 \times 900$. The results for $\delta=0, Q=0.5$ and different reaction rates are shown in Fig. 8. If $\lambda=16, \lambda^{\prime}=8$, then the non-universal term in Eq. (50) is zero and $\alpha \approx 0.22$ as in the case of infinite reaction rates. This is consistent with dashed line in Fig. 8 being parallel to the solid line. If however, $\lambda=8, \lambda^{\prime}=8$, then $\alpha \approx-0.57$. The slope of the bottom line in Fig. 8 is clearly negative.

\section{SUMMARY AND CONCLUSIONS}

In summary, we calculated the large time behavior of the survival probability of a test particle in a system of coagulating and annihilating random walkers in $d \leq 2$. In one dimension, this generalizes the site persistence problem in the $q$-state Potts model evolving via zero temperature Glauber dynamics. The survival probability was shown to decay as a power law. In $d<2$, the exponent $\theta$ characterizing this power law was shown to be univer- sal, in the sense that it depends only on $\delta$ and $Q$ and was independent of $\lambda, \lambda^{\prime}$. The renormalization group formalism provided a systematic way of calculating the survival probability for the entire parameter space.

In two dimensions, we computed the logarithmic corrections to the power law decay. It was shown that to compute the correct logarithmic factors, one had to include contributions from one-loop diagrams and not just the tree level diagrams as was done in earlier work. The behavior of the survival probability in two dimensions is surprising. First, the power law decay is universal and thus does not coincide with the rate equation result, even though $d=2$ is the upper critical dimension. Second, the logarithmic corrections to the power law are non-universal and depend on the reaction rates. This is contrary to the general expectation that kinetics of reaction-diffusion systems are diffusion limited below the upper critical dimension. Both the universality of the power law and the non-universality of log-corrections in two dimensions can be traced to the fact that the rate equation exponent is given by the ratio of microscopic rates, which are both marginally relevant in two dimensions.

From the renormalization group point of view, by studying the logarithmic derivative of the mean density of $B$-particles, we have considerably simplified the schemes used in Refs. 2, 6, 9]. While the exponents are only calculated up to first order in $\epsilon$, the renormalization group method remains the only systematic way of computing the exponents when an exact solution is not available.

\section{Acknowledgments}

RR would like to acknowledge support from NSF grant DMR-0207106.
[1] B. Derrida, V. Hakim, and V. Pasquier, Phys. Rev. Lett. 75, 751 (1995).

[2] M. J. Howard, J. Phys. A 29, 3437 (1996).

[3] C. Monthus, Phys. Rev. E 54, 4844 (1996).

[4] S. N. Majumdar and S. J. Cornell, Phys. Rev. E 57, 3757 (1998).

[5] S. J. O'Donoghue and A. J. Bray, Phys. Rev. E 65, 051114 (2002).

[6] S. Krishnamurthy, R. Rajesh, and O. Zaboronski, Phys. Rev. E 68, 046103 (2003).

[7] S. Cueille and C. Sire, Phys. Rev. E 55, 5465 (1997).

[8] E. K. O. Hellén, P. E. Salmi, and M. J. Alava, Europhys. Lett. 59, 186 (2002).

[9] S. Krishnamurthy, R. Rajesh, and O. Zaboronski, Phys. Rev. E 66, 066118 (2002).

[10] S. Redner and P. L. Krapivsky, Am. J. Phys. 67, 1277 (1999).

[11] J. Cardy and M. Katori, J. Phys. A 36, 609 (2003).

[12] M. Smoluchowski, Z. Phys. Chem. 92, 215 (1917).

[13] S. Chandrasekhar, Rev. Mod. Phys. 15, 1 (1943).
[14] L. Peliti, J. Phys. A 19, L365 (1986).

[15] B. P. Lee, J. Phys. A 27, 2633 (1994).

[16] J. Cardy, J. Phys. A 28, L19 (1995).

[17] P. L. Krapivsky, E. Ben-Naim, and S. Redner, Phys. Rev. E 50, 2474 (1994).

[18] M. E. Fisher and M. P. Gelfand, J. Stat. Phys. 53, 175 (1988).

[19] M. Doi, J. Phys. A 9, 1465 (1976).

[20] M. Doi, J. Phys. A 9, 1479 (1976).

[21] Y. B. Zel'dovich and A. A. Ovchinnikov, Soviet Phys. JETP 47, 829 (1978).

[22] J. Cardy, Field theory and nonequilibrium statistical mechanics, notes available at the website http://www-thphys.physics.ox.ac.uk/users/JohnCardy/

[23] C. Itzykson and J. M. Drouffe, Statistical Field Theory, Cambridge monographs on mathematical physics (Cambridge University Press, Cambridge, 1994).

[24] R. Rajesh and O. Zaboronski, unpublished. 